\title{
Physics
}

\section{in the middle of the Balkans}

n Alexander G. Petrov, Nicholay S. Tonchev and Oleg I. Yordanov - DOI: 10.1051/epn/2011602

\author{
Half a century ago, the Bulgarian Physics community created the Union of the \\ Physicists in Bulgaria (UPB), as in many other countries in that post war period. \\ In this article we outline the history of Physics in Bulgaria, its current state and \\ discuss its future place and perspectives within the Balkan region and in Europe.
}

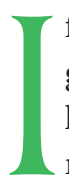

f founded formally in 1971, UPB has a much longer and illustrious history. Twenty years after the liberation from the Ottoman rule, the physicists and mathematicians founded the Bulgarian Physical and Mathematical Society (BPMS, Sofia, March 29, 1898). Note that the secular education with limited mathematics came into the Bulgarian schools as late as in the 1830s-40s, the first Bulgarian high school starting in the early 1860s. The first higher education institution arrived as a pedagogical school in 1888 to become in 1904 the Sofia University (SU).

Facing more and more students and professionals beyond 1950, BPMS split (1971) into Bulgarian Physical Society and Bulgarian Mathematical Society. On November 25, 1989, two months after the fall of the Berlin wall, the Bulgarian Physical Society reorganized and became the Union of the Physicists in Bulgaria.

Independent of political vagaries, the presidents of UPB or previous organizations have always been outstanding physicists. In the difficult 1945-1950 period the president was Georgi Nadjakov, Fellow of the Bulgarian Academy of Sciences (FBAS), who had worked with both Marie Curie and Paul Langevin in the 20-ies. His discovery of the photo-electret state of matter led to the photocopier invention by Chester Carlson. One of the Institutes of the BAS bears Nadjakov's name. We can also cite Christo Christov, FBAS, president of BPS from 1971 to 1986, and Milko Borissov, FBAS (1986-1989). The first UPB president was Ivan Zlatev, until 1992, succeeded by Ivan Lalov who, during his term (1992-2001), served for a short period as Minister of the Bulgarian Ministry of Education, Science and Technologies. Matey Mateev, FBAS, succeeded him in 2001 until his sudden death in 2010. During Mateev's term as Minister of Education and Science in the early nineties, the first law organizing the democratic education was prepared and successfully passed by the Bulgarian Parliament. In the latest 7th Regular UPB Congress, held in April 2011, one of the authors of this article, A.G.Petrov, FBAS, was elected UPB President for the next three years term.

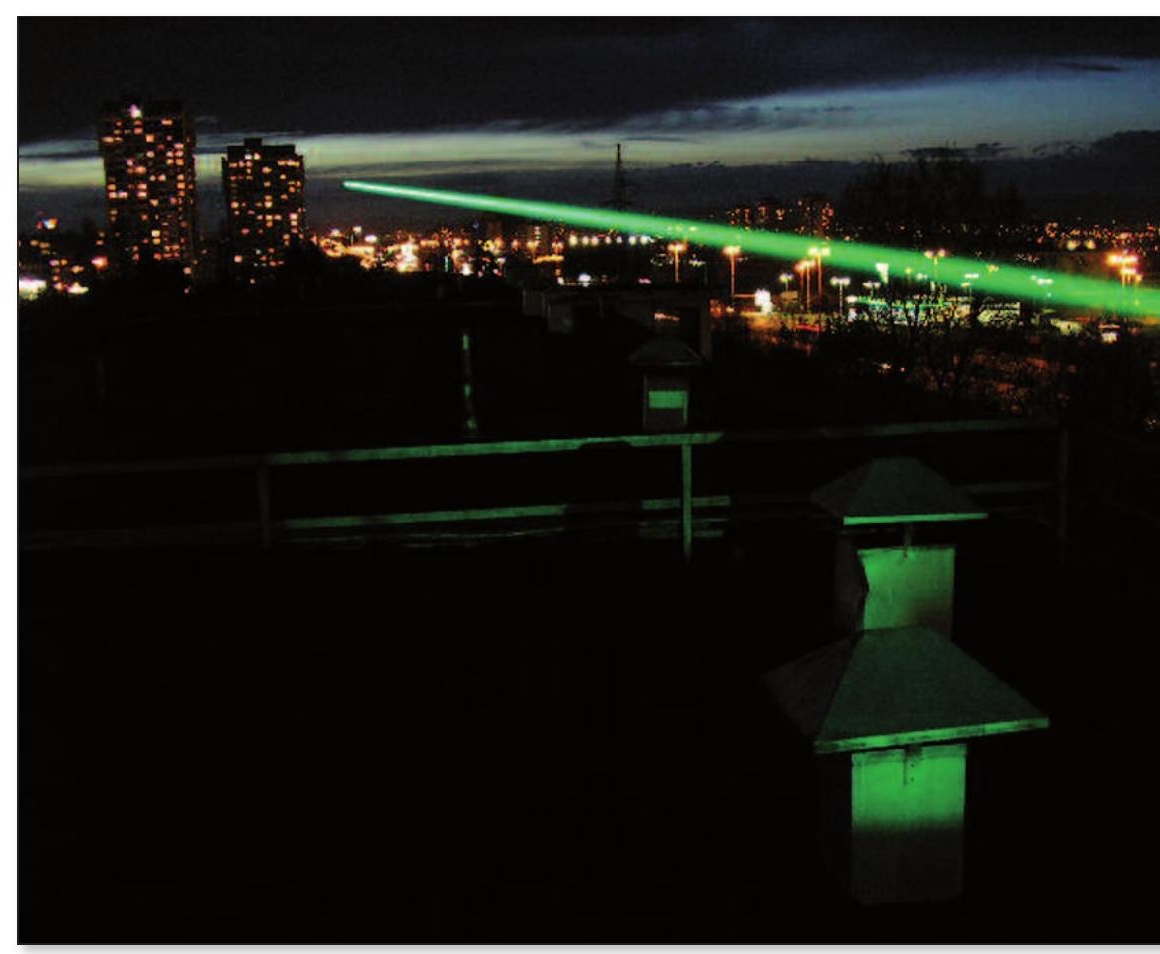

Current state of Physics, Physics education in Bulgaria and UPB activities Today, UPB has a membership of over 400, among 100 of whom active. The mission of UPB is to support and coordinate the activities of its members in fundamental and applied research, industry, medical physics, environmental protection, secondary and university education in physics and to popularize the achievements in physical sciences. With an annual budget of only $7500 €$, the Union supports 16 regional branches in virtually all major Bulgarian cities. The funding comes from the Kozloduy nuclear power plant, Nuclear Regulation Agency of Bulgaria, Ministry of Education and Science and private companies. Majors in Physics are offered in 4 of the Bulgarian higher schools: Sofia (258 students), Plovdiv (157), Blagoevgrad (68) and Shumen (46) universities. The number of graduates in 2010, however, was only 185 . $\triangle$ FIG. 1:

The high power CuBr-vapor lase beam over the skyline of Sofia during the 2005 laser show staged by the Union of Physicists in Bulgaria to celebrate the World Year of Physics.

The laser is operating from the roof of the Institute of

Electronics, Bulgarian Academy of Sciences. 
There were $40 \mathrm{PhDs}$ the same year. In addition, chairs of Physics are established in all technical and medical schools, which brings the number of students exposed to some form of Physics education to 1539 . Considering also the reduced share of Physics in the curriculum of the elementary and secondary schools, its current impact on society is unacceptable for the community. Our efforts are towards changing that.

The other troublesome issue is the extremely low funding for research and instruction. The 7 physics institutes of BAS together receive 3.89 M€ of state subsidy to which 2.2 $\mathrm{M} €$ are added through various projects. The state subsidy for university physics is estimated to be $1.26 \mathrm{M} €$ plus 0.5 $\mathrm{M} €$ from projects. This sums up to a miserable $7.85 \mathrm{M} €$ all together in 2010. The part of the GDP devoted to science in Bulgaria is not only the lowest in EU but also lower than in many third world countries. Bulgaria is the only European country that did not secure support for science from the EU structural funds. This unfortunate situation, discussed recently in Nature ${ }^{1}$ was since further aggravated by the widespread mismanagement of the science funding by the government bureaucrats ${ }^{2}$. The low government funding for science results in a limited number of science-involved firms and to a negligible private funding for science. This vicious circle could be cut out either by a conceptually different government policy or by external pressure. The Bulgarian scientists fought back by mobilizing the international community: many colleagues, among them nine Nobel prize winners and six Fields medallists signed the petition in defence of the Bulgarian science, www.science.nauka2010.com Especially active in voicing their support was the European Physics Community to whom we extend our hearty gratitude. UPB organizes or endorses a number of conferences in Bulgaria each year. One of the major events run by UPB is the Annual Spring National Conference on Physics Education. In its 3 days programme, it covers: up-to-date educational issues, methodology of teaching, classroom physics experiments and student presentations. Each conference has its own focus, for example, for the $39^{\text {th }}$ this year, it was the $100^{\text {th }}$ Anniversary of the Rutherford Model.

\section{Reaching out to the Balkan region and to Europe.}

UPB is one of the founders and active member of the Balkan Physical Union (BPU), created in 1985 as a nonprofit organization without any governmental sponsorship. The aim is to promote collaborations and joint research projects between the countries in the Balkan region in all fields of Physical sciences. Many conferences, workshops and schools have been organized by the national physical societies of the Balkan countries under the banner of the BPU. The leading event is the General conference of the BPU, which, according to the BPU Constitution, is to convene every three years consecutively in different countries. The first was in Greece (1991). The $4^{\text {th }}$ held in Bulgaria (2000) brought up 800 attendees presenting 750 oral or poster communication. The last took place in Greece in 2009: over 450 papers were published in a 1500 pages volume (No. 1203 of AIP Conference Proceedings). The next, $8^{\text {th }}$ conference, will be in Romania (2012).

The collaboration between UPB and EPS sizably intensified during the last two decades and a lot more could and should be done. Two major EPS events took place in Bulgaria: the $23^{\text {rd }}$ meeting of the European Group on Atomic Spectroscopy (2002) and the $36^{\text {th }}$ EPS Conf. on Plasma Physics (2009) to which 558 colleagues (103 students) attended. The young Bulgarian physicists welcomed the 46 EPS grants provided between 2001 and 2007 to attend various European events. UPB participated to several EPS initiatives: the laser show of the 2005 Year of Physics and the European scientists' night in 2008, 2009 and 2010.

UPB publishes two Physics journals, the professional, peer reviewed, Bulgarian Journal of Physics (BJP, papers in English) and the more popular World of Physics. We take the present opportunity to invite members of EPS to submit contributions to BJP in all branches of Physics.

\section{About the Authors}

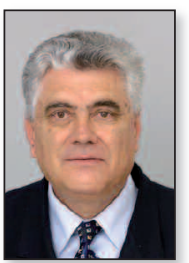

Professor Alexander G. Petrov, $\mathrm{PhD}$ (1974), DSc(1987), FBAS, is Director of the Institute of Solid State Physics (ISSP) at the Bulgarian Academy of Sciences (BAS) and President of UPB. He has authored over 175 papers and a monograph, The Lyotropic State of Matter, Gordon and Breach, NJ-L (1999). He is a recipient of the Freedericksz Medal of the Russian Liquid Crystal Society (2005) and of the Prize for Outstanding Contribution to Science in Bulgaria (2007).

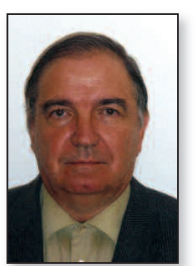

Professor Nicholai S. Tonchev, PhD (1976), DSc (1986) is Chairman of the Scientific Council of ISSP at BAS. He has authored over 100 papers and is a coauthor of two monographs: "The Approximating Hamiltonian Method in Statistical Physics" (in Russian), Publ. House of BAS, Sofia (1981) and "Theory of Critical Phenomena in FiniteSize Systems; Scaling and Quantum Effects”, World Scientific (2000). He is Editor-in-Chief of the Bulgarian Journal of Physics and Scientific Secretary of the BPU.

Dr. Oleg I. Yordanov is Head of the Laboratory of Micro-

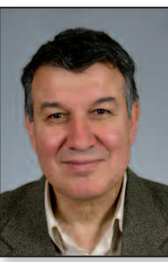
wave Physics and Technologies, Institute of Electronics, BAS. His MSs (1977, SU) was in field of Mathematical Physics; his $\mathrm{PhD}$ thesis (1991, BAS) was on scattering of EM waves from rough surfaces. He is an Editor of the World of Physics. 